\title{
A bacterial ATP-dependent, enhancer binding protein that activates the housekeeping RNA polymerase
}

\author{
William C. Bowman and Robert G. Kranz ${ }^{1}$ \\ Department of Biology, Washington University, St. Louis, Missouri 63130 USA
}

\begin{abstract}
A commonly accepted view of gene regulation in bacteria that has emerged over the last decade is that promoters are transcriptionaly activated by one of two general mechanisms. The major type involves activator proteins that bind to DNA adjacent to where the RNA polymerase (RNAP) holoenzyme binds, usually assisting in recruitment of the RNAP to the promoter. This holoenzyme uses the housekeeping $\sigma^{70}$ or a related factor, which directs the core RNAP to the promoter and assists in melting the DNA near the RNA start site A second type of mechanism involves the altemative sigma factor (called $\sigma^{54}$ or $\sigma^{N}$ ) that directs RNAP to highly conserved promoters. In these cases, an activator protein with an ATPase function oligomerizes at tandem sites far upstream from the promoter. The nitrogen regulatory protein (NtrC) from enteric bacteria has been the model for this family of activators. Activation of the RNAP/ $\sigma^{54}$ holoenzyme to form the open complex is mediated by the activator, which is tethered upstream. Hence, this class of protein is sometimes called the enhancer binding protein family or the NtrC class. We describe here a third system that has properties of each of these two types. The NtrC enhancer binding protein from the photosynthetic bacterium, Rhodobacter capsulatus, is shown in vitro to activate the housekeeping RNAP/ ${ }^{70}$ holoenzyme. Transcriptional activation by this NtrC requires ATP binding but not hydrolysis. Oligomerization at distant tandem binding sites on a supercoiled template is also necessary. Mechanistic and evolutionary questions of these systems are discussed.
\end{abstract}

[Key Words: Gene regulation; enhancer; ATP-dependent; housekeeping hol oenzyme]

Received December 23, 1997; revised version accepted A pril 16, 1998.

Compilations of data from studies on nearly 150 regulated promoters in enteric bacteria have led to the view that a bacterial promoter is activated by use of one of two systems (for review, see Collado-Vides et al . 1991; Gralla and Collado-Vides 1996). These systems are denoted by where the activator protein binds on the DN A and which sigma factor is used, but a key feature is that the mechanism of activation is quite different for each. The first system involves the RNA polymerase (RNAP) containing the $\sigma^{70}$ factor. All bacterial sigma factors are related to this housekeeping sigma (except sigma 54, see below) (Lonetto et al. 1992). In these cases, the activator proteins bind between 30 and 80-bp upstream of the transcription start site, adjacent to the RNAP holoenzyme. These promoters are usually poorly recognized by RN AP without the aided recruitment by the activator protein (Ishihama 1993). Many el egant studies over the last decade have defined the exact residues of specific activators and of the RNAP subunits that make contact with each other. So far, interactions with either the $\alpha$ and/or $\sigma$ subunits of RN AP have been characterized (for review,

${ }^{1}$ Corresponding author.

E-MAIL Kranz@biodec.wustl.edu; FAX (314) 935-4432. see Busby and Ebright 1994). On the basis of these studies, certain rules are beginning to emerge with respect to which RNAP contact is made according to where activator binding is centered (between -30 and -80 ).

A second general type of bacterial activation system involves RN AP containing the rpoN-encoded sigma factor called $\sigma^{54}\left(\sigma^{N}\right)$ (for review, see M errick 1993; N orth et al. 1993; Magasanik 1996). This is the only sigma factor that is unrelated to $\sigma^{70}$ and it is responsible for directing RNAP to very highly conserved promoters that have the consensus G G-N ${ }_{10}-\mathrm{GC}$ (at -24 and -12 bp). In these cases the activator proteins bind distantly upstream of the promoters and via DNA looping, activate the RNAP/ $\sigma^{54}$ holoenzyme. A paradigm for this class of activator, for which there are now at least 30 cases in many genera of bacteria, is the nitrogen regulatory protein called $\mathrm{NtrC}$ (or NRI). Members of the N trC class typically bind to DN A at tandem sites centered $>100$-bp upstream of the start site and these activators are therefore referred to as enhancer-binding proteins (EBPs). In addition to the $\sigma^{54}$ and enhancer characteristics, all EBPs contain a nucleotide binding fold for which its ATPase function is required for transcriptional activation (Weiss et al. 1991; Austin and Dixon 1992). For some of the EBP-activated 
promoters, RNAP/ $\sigma^{54}$ has been shown to bind the promoter in a stable closed complex, independent of the activator, indicating that recruitment to the promoter is not necessary (Ninfa et al. 1987; Sasse-Dwight and Gralla 1988; Buck and Cannon 1992; Cannon et al. 1993; Syed and Gralla 1997). For N trC, an unusual multimerization occurs whereby two dimers bind at the tandem sites and then two more di mers bind to these by proteinprotein interactions (Wyman et al . 1997). This DN A-mediated oligomerization is necessary for ATPase activity and activation, perhaps prompting an interaction with both the $\sigma^{54}$ and $\beta$ subunits of RNAP (Lee and Hoover 1995).

Here, we describe a third bacterial activation system with properties of both systems. Like the enteric counterpart (N infa and Magasanik 1986), the Rhodobacter capsulatus $\mathrm{N}$ trC protein ( $\mathrm{RcN} \operatorname{trC}$ ) is phosphorylated by its cognate kinase, $\mathrm{NtrB}$, in response to nitrogen deprivation (Cullen et al. 1996). This phosphorylation in enterics converts $\mathrm{N}$ trC into the activator with the aforementioned characteristics. The RcNtrC also contains the nucleotide binding fold, which, by genetic analysis, is required for its activation function in vivo (FosterHartnett et al. 1994). T wo tandem sites that are centered $>100$-bp upstream of the transcription start site bind purified $\mathrm{RCN}$ trC in each of the five known members of the RcN trC regulon (nifA 1, nifA 2, glnB, anfA, and mopA). The upstream tandem sites are necessary for activation in vivo, as demonstrated with the nifA 1 and nifA 2 promoters (Foster-Hartnett et al. 1994). However, RcN trC- activated promoters are still expressed in a nitrogen-dependent manner in rpoN mutants and they have no sequence similarity to typical $\sigma^{54}$-activated promoters (Foster-Hartnett and Kranz 1992, 1994; Preker et al. 1992; Kutsche et al. 1996). Thus, the RN AP holoenzyme that is activated by the RcN trC protein has remained an enigma. Here, we show by use of purified components that the R. capsulatus RNAP (RCRNAP) $/ \sigma^{70}$ holoenzyme is activated by RcNtrC in an N trB-dependent manner. In vitro transcriptional activation requires a supercoiled template, the upstream tandem binding sites for RcNtrC, and ATP. Our results suggest that this third type of system contains elements of each of the first two and could represent an evolutionary transition between $\sigma^{54}$ and $\sigma^{70}$ systems that were retained by this $\alpha$ proteobacterial species of photosynthetic bacterium.

\section{Results}

RcNtrC activates the RNAP $\sigma^{70}$ holoenzyme in an NtrB-dependent manner

To investigate whether the RcRNAP $\sigma^{70}$ holoenzyme is activated by the RcN trC system, we have combined the use of a set of wild-type and mutant nifA 1 promoters (Fig. 1A) and hyperactive constitutive RcN trC mutant proteins. These RcN trC proteins show increased in vivo activation in response to limiting or sufficient nitrogen. It is known that they are phosphorylated in vitro by $\mathrm{N}$ trB

A

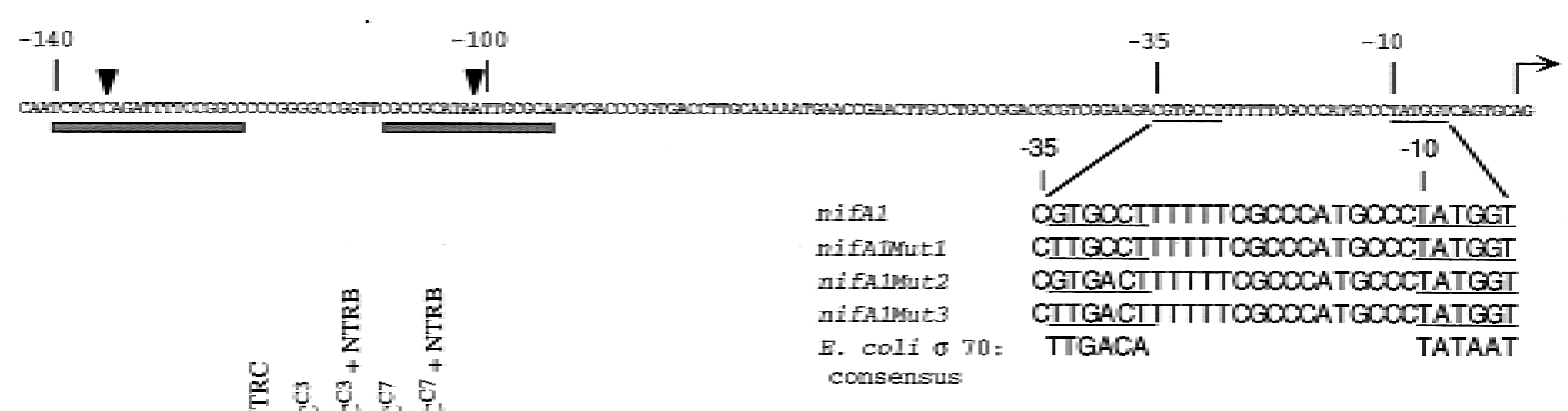

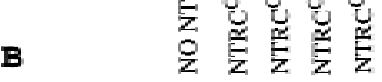

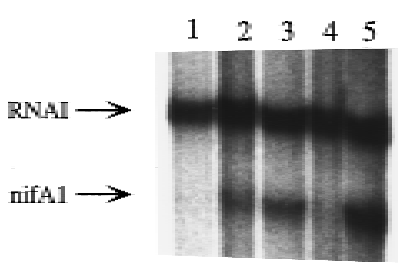

nifA1

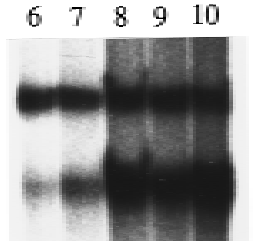

nifA1Mut 1

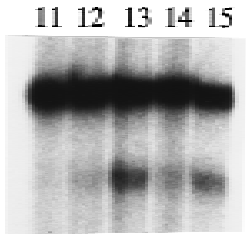

nifAlMur2

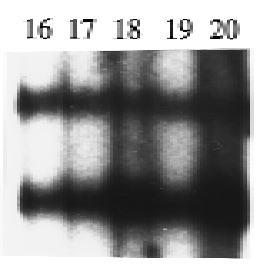

nifA1Mut3

Figure 1. (A) Sequence of the nifA 1 promoter region and engineered promoters. (Shaded bars) RcN trC binding sites, (arrowheads) major DN ase I hypersensitive sites. The -35 and -10 hexamers are underlined and the transcriptional start site is marked by a horizontal arrow. The sequences of the nifA 1, nifA 1Mut1, nifA 1Mut2, and nifA 1Mut3 promoters are al igned for comparison with the E. coli $\sigma^{70}$ consensus. (B) In vitro transcriptional activation of nifA 1 wild-type and mutant promoters by RcN trC. Templates are noted bel ow each set of transcription reactions. (Lanes 1,6,11,16) No activators; (lanes 2,7,12,17) $260 \mathrm{~nm} \mathrm{RcN}_{\text {trC }} \mathrm{C}^{3} ;(\mathrm{lanes} 3,8,13,18) 260 \mathrm{nM}$ RcN trC ${ }^{\mathrm{C3}}$ and 270 nм MBP-NTRB; (lanes 4,9,14,19) 350 nм RcN trC ${ }^{\mathrm{C} 7}$; (lanes 5,10,15,20) 350 nm RcN trC ${ }^{\mathrm{C7}}$ and $270 \mathrm{~nm}$ M BP-N trB. 
to the same level and bind to the upstream tandem sites with the same affinity as the wild-type RcN trC (W.C. Bowman, P.J. Cullen, and R.G. Kranz, unpubl.). For in vitro transcription studies, we have recently purified the R. capsulatus housekeeping RN AP contai ning the major $\sigma^{70}$ subunit. This preparation is $\sim 98 \%$ pure by SDSPAGE and contains only the housekeeping $\sigma^{70}$ as determined by Western blot analysis with the $2 \mathrm{G} 10$ monoclonal antibody (Cullen et al . 1997) (Fig. 2A,B). We chose the nifA 1 promoter because of the in vivo information already known about its upstream DNA including its RcNtrC tandem binding sites (Foster-Hartnett et al. 1994). Moreover, we previously converted the nucleotides in the -35 region of nifA 1 toward an ideal -35 hexamer (nifA1Mut3) with the result of high-level basal transcription by the $\sigma^{70}$ RN AP (Cullen et al. 1997). Thus, it was known that with only two nucleotide changes, the RCRNAP $\sigma^{70}$ could melt this promoter by an RcN trCindependent process.

For the present study, we converted the wild-type

A

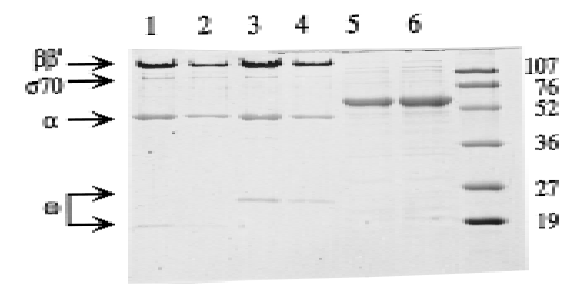

B

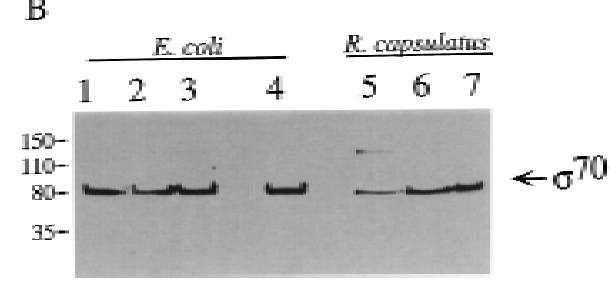

C

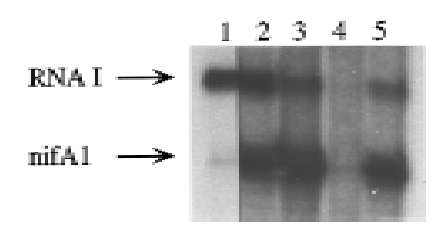

Figure 2. Characterization of the holoenzyme and activator preparations and transcription inhibition by monoclonal antibody 2G10. (A) SDS-PAGE of the E. coli RNAP (Ianes 1,2); RcRN AP (lanes 3,4); RcN trC ${ }^{\mathrm{C3}}$ (lane 5) and RcN $\operatorname{trC}^{\mathrm{C7}}$ (lane 6); standards with sizes indicated in KD. (B) Western analysis of the RcRNAP and EcRNAP purification by use of $m A b 2$ G10, specific for $\mathrm{E}$. coli $\sigma^{70}$. Fractions are from E. coli and R. capsulatus as follows: (Lanes 1,5) PEG precipitation fraction; (lanes 2,6) heparin agarose fraction; (lanes 3,7) DEAE-Sepharose fraction; (lane 4) HPLC-purified EcRN AP/ $\sigma^{70}$ holoenzyme. (C) RcN trC activated transcription is abolished by $\sigma^{70} \mathrm{mAb} 2 \mathrm{G} 10$. The nifA 1Mut1 promoter was used. (Lane 1) no activator; (lanes 2-5) 350 nM RcN trC ${ }^{\text {C7 }}$; (lanes 3-5) 270 nM M BP-N trB; (lane 4) $1 \mu$ of $\mathrm{mAb} 2 \mathrm{G} 10$; (lane 5) $1 \mu \mathrm{l}$ of control mAb.
nifA 1 promoter toward a consensus -35 hexamer by one (nifA 1M ut1, nifA 1M ut2) or two nucleotides (nifA 1M ut3) and studied transcription in the presence of purified maltose binding protein-N trB (MBP-N trB) and two RcNtrC constitutive mutant proteins, designated $\mathrm{RcN} \operatorname{trC}^{\mathrm{C} 3}$ and $\mathrm{RcN} \operatorname{trC}^{\mathrm{C} 7}$. These proteins were purified as described previously (Cullen et al. 1996) to $>95 \%$ homogeneity (Fig. 2A). All transcription reactions shown in Figure 1 were with supercoiled templates in which the indicated nifA1 DNA was cloned upstream of a transcriptional terminator. nifA 1 transcription yields an mRNA product of 92 nucleotides that migrates below the 108-nucleotide RNAI transcript. The RNAI transcript is from a well-recognized $\sigma^{70}$-type promoter (Scott 1984) that functions with both the Escherichia coli and RcRNAP holoenzymes (Cullen et al. 1997). RN Al transcripts serve as internal controls for each template and all nifA 1 transcripts detected in the present study were quantified relative to the RNAI transcript. This corrected for loading differences or degradation and facilitated comparisons with different templates or conditions. In the absence of activator the nifA1Mut3 template exhibited high transcription (Fig. 1B, lane 16). Whereas nifA1Mut1 (Fig. 1B, lane 6) gave a very low basal level of transcription, the wild-type (Fig. 1B, lane 1) and nifA 1Mut2 (Fig. 1B, lane 11) exhibited no detectable transcription without activation. Addition of the RcN trC proteins resulted in at least a 4- to 10-fold increase in transcription at the nifA 1 wild-type, nifA 1M ut1, and nifA 1Mut2 promoters (Fig. 1B). Because no basal level of transcription could be detected with the wild-type or nifA1Mut2 promoters, the fold increase could not be determined. However, at least 10-fold less product from the activated sample could have been detected, indicating at minimum, a 10-fold activation. Because basal levels are so high, activated transcription of the nifA 1Mut3 promoter is obscured (Fig. 1B, lanes 1620). No increase in transcription was observed at the RNAI promoter on addition of the activators, indicating that the activation was specific for nifA 1 and the two mutant promoters. The addition of MBP-N trB to reactions containing each of the three promoters (wild type, nifA 1Mut1, and nifA 1Mut2) and RcN trC increased the activation another two- to fourfold, depending on the promoter or RcNtrC allele. However, total activation levels were al ways higher when the nifA 1M ut1 promoter was used, at least fivefold higher than observed with the wild-type and nifA 1Mut2 promoters. To define the activation requirements for this system, results with the nifA 1M ut1 promoter are presented here, although most activation requirements were also demonstrated with the wild-type nifA 1 promoter.

With nifA1Mut1, the RcNtrC ${ }^{\mathrm{C} 7}$ protein gives the greatest activation without MBP-N trB (Fig. 1B, lane 9) and total activated transcription with MBP-N trB (Figs. 1B, lane 10, and 2C, lanes 2,3). Previously, we have used the $\sigma^{70}$ monoclonal antibody 2G10 to inhibit transcription of RCRN AP $\sigma^{70}$ promoters as have other groups with E. coli $\sigma^{70}$ promoters (for review, see Breyer et al. 1997 and references therein). In vitro transcription of both the 
RNAI promoter and the activated nifA 1Mut1 promoter is inhi bited $>98 \%$ by $\mathrm{mAb} 2 \mathrm{G} 10$ (Fig. $2 \mathrm{C}$, lane 4 ) but not significantly by a control mAb (Iane 5). The 2G10 antibody was also found to inhibit RcN trC-dependent transcriptional activation of the wild-type nifAl promoter (data not shown). The only sigma factor in the RcRN AP preparation that reacts with $2 \mathrm{G} 10$ is $\sigma^{70}$ (Cullen et al. 1997; Fig. 2B, lane 7). The epitope for 2G10 has been mapped to residues $470-486$ of the E. coli $\sigma^{70}$ factor (Breyer et al. 1997). As expected, the R. capsulatus $\sigma^{70}$ is highly conserved in this region (Pasternak et al. 1996), but no $\sigma^{54}$ factor, including R. capsulatus $\sigma^{54}$ Jones and Haselkorn 1989), contain this epitope.

To further demonstrate that RcN trC activates the R. capsulatus $\sigma^{70}$ holoenzyme, we constructed two plasmids containing the $R$. capsulatus rpoD gene that result in overexpression of the R. capsulatus $\sigma^{70}$ subunit in $E$. coli. The $\sigma^{70}$ protein expressed from pRGK300 was purified from an SDS gel and renatured by use of the method of Gross et al. (1978). A hexahistidine-tagged $\sigma^{70}$ polypeptide was overexpressed from pRGK301 and purified with a nickel affinity column. The purified $\sigma^{70}$ proteins were added to in vitro transcription reactions containing R. capsulatus core RNA polymerase that has been shown previously to contain very low levels of $\sigma^{70}$ (Cullen et al. 1997). This core preparation gave very low levels of RNAI transcript and no nifA 1Mut1 transcript with or without RcN tr proteins (Fig. 3, lanes 1, 2). Addition of either the histidinetagged (Fig. 3, lanes 3,4) or the renatured $\sigma^{70}$ (Fig. 3, lane 5) resulted in a >10-fold increase in the RNAl transcript. When $\mathrm{N} \operatorname{trB}$ and $\mathrm{N} \operatorname{trC}$ was added to either reaction containing $\sigma^{70}$ (Fig. 3, lane $4,5)$ a significant increase in activation is observed compared with the reaction with core RNAP and $\sigma^{70}$ only (Fig. 3, lane 3). These results confirm that it is the R. capsulatus $\sigma^{70}$ RN AP that is activated by RcN trC.

It is worth noting the presence of a subunit of the RCRNAP preparation, which migrates at $\sim 23 \mathrm{kD}$ in this SDS-PAGE system (Fig. 2A, lane 3). The E. coli holoenzyme preparation contains a polypeptide that migrates $\sim 4 \mathrm{kD}$ smaller then this (Fig 2A, lane 1), which is similar in size to the previously noted $\omega$ subunit of unknown function (Gentry and Burgess 1986). Amino-terminal sequencing of the $R$. capsulatus 23-kD polypeptide has demonstrated that it is the $\omega$ subunit. The R. capsulatus

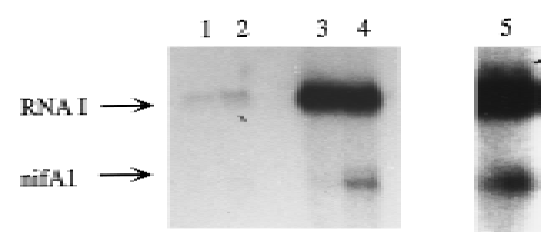

Figure 3. Purified $\sigma^{70}$ added to R. capsulatus core RNAP stimulates transcriptional activation by $\mathrm{RcN}$ trC. In vitro transcription reactions using the supercoiled nifA 1Mut1 template with the R. capsulatus core RNAP (lanes 1,2) or core RNAP with the addition of His-tagged $\sigma^{70}$ subunit (lanes 3,4$)$ or SDSgel purified $\sigma^{70}$ subunit (lane 5). (Lanes 1,3) no activator; (lanes $2,4,5) 270$ nM MBP-N trB and 260 nM RcN trC ${ }^{\mathrm{C} 3}$. gene (rpoZ) encoding the $\omega$ subunit has been sequenced (R. capsulatus sequencing project at the University of Chicago, URL http://capsulapedia.uchicago.edu). The rpoZ gene sequence indicates a size that is 28 residues larger than the E. coli counterpart, consistent with the size exhibited by SDS-PAGE.

Supercoiled DNA and both upstream tandem sites are required for RcNtrC-mediated activation

To determine if DNA binding by RcN trC is required for activation, and, if so, whether one dimer site or two are necessary, we constructed plasmids in which either one or both of the RcN trC binding sites have been removed from the nifA 1M ut1 promoter region. The singl e binding site that was removed was site I, the furthest upstream. This site has been shown to be necessary for in vivo activation (Foster-Hartnett et al. 1994) and deletion of it maintains the sequence/structure of DN A between site II and the promoter. Even on supercoiled templates, removal of one or both RcNtrC binding sites completely abolishes transcriptional activation of the nifA 1Mut1 promoter by the RcN trC proteins (Fig. 4). Basal transcription from the supercoiled templates is unaffected by deletion of the binding sites. No activation was observed with either of the binding site del etion templates when the RcN trC concentration was increased to $875 \mathrm{~nm}$ (data not shown). These data show that the RcN trC protein must bind DNA to activate transcription and suggest that there is a cooperative interaction between the bound RcN trC dimers that is also necessary.

Previously, we have shown that phosphorylation of the RcN trC protein leads to a fourfold increase in binding to the tandem sites located upstream from the glnB promoter (Cullen et al. 1996). We have also shown that unphosphorylated RcN trC still binds upstream from the nifAl promoter when one of the two tandem sites has been removed, although approximately fourfold more RcN trC is required (compared with the binding at tandem sites) (Foster-Hartnett et al. 1994). To determine whether phosphorylation increases the affinity of RcN trC for a single site, DN ase I footprinting was performed with $\mathrm{RcN} \mathrm{trC}^{\mathrm{C} 3}$ in the presence and absence of MBP-N trB by use of the EcoRI-HindlII fragment from pALB1, the template that contains a single RcN trC binding site, as a probe. The concentration of unphosphorylated and phosphorylated $\mathrm{RcN} \operatorname{trC}^{\mathrm{C} 3}$ that protected from DN ase I digestion was found to be $160 \mathrm{~nm}$ and $80 \mathrm{~nm}$, respectively (data not shown). These results support the idea that transcriptional activation is dependent on a cooperative interaction that occurs between RcNtrC dimers when they are bound to the two tandem sites (see Discussion).

When the nifA1Mut1 template with intact tandem binding sites was linearized, no transcriptional activation by RcN trC \pm M BP-N trB was detected (Fig. 4, lanes 16-20). Under these conditions, much less nifA 1Mut1 and RNAI basal transcription was observed. Cut and re circularized template also showed no transcriptional activation by RcN trC, which is consistent with the obser- 


\section{Bowman and Kranz}

Figure 4. RcN trC-activated transcription is only observed on supercoiled templates with intact tandem binding sites. In vitro transcription reactions were performed using supercoiled (lanes 1-15) or linear templates (lanes 16-20) that contained 0 (lanes 11-15), 1 (lanes 6-10), or both (Ianes 1-5; 16-20) tandem RcNtrC dimer binding sites. (Lanes 1,6,11,16) No activators; (lanes2,7,12,17) 260 nM RcN trC ${ }^{\text {C3; }}$; (lanes $3,8,13,18) 260 \mathrm{~nm} \mathrm{RcN} \mathrm{trC}^{\mathrm{C} 3}$ and $270 \mathrm{~nm}$ MBP-NTRB; (lanes 4,9,14,19) 350 nM RcN trC ${ }^{\mathrm{C7}}$; (lanes 5,10,15,20) $350 \mathrm{~nm}$ $\mathrm{RcN} \operatorname{trC}^{\mathrm{C} 7}$ and $270 \mathrm{~nm} \mathrm{MBP}-\mathrm{N}$ trB.

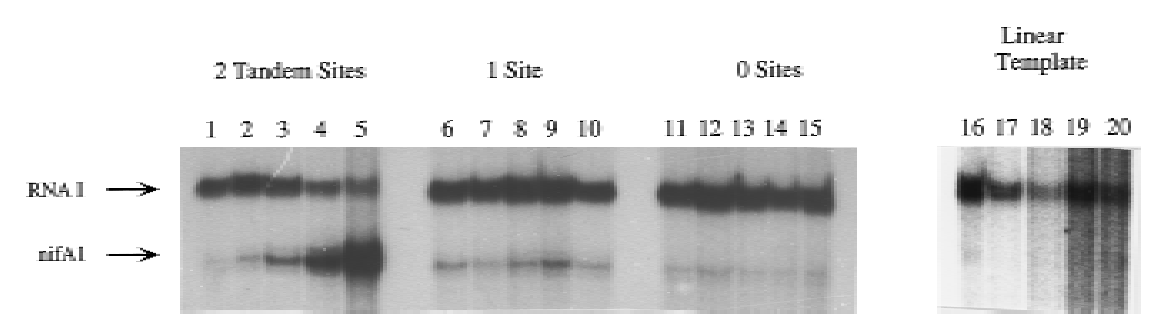

vation that an increase in nicked template results in poorer transcriptional activation by RcN trC (data not shown).

ATP binding, but not hydrolysis, is necessary for RcNtrC to activate transcription

To determine whether ATP hydrolysis is necessary for transcriptional activation by $\mathrm{RcN}$ trC, in vitro transcriptional activation reactions were performed by use of the $\beta-\gamma$ nonhydrolyzable ATP anal og adenylyl imidodiphosphate (AM P-PN P). Because transcri pti on requires hydrolysis of the $\alpha-\beta$ bond, AM P-PN P can be utilized by the RNA polymerase, but not by RcN trC for an ATPase activity. This analog has been used previously to investigate the ATPase function for activation by enteric N trC (e.g., Wang et al. 1995; Syed and Gralla 1997). The $\mathrm{RcN} \operatorname{trC} \mathrm{C}^{\mathrm{C}}$ protein was used because of its high level of activation that is independent of $\mathrm{MBP}-\mathrm{N}$ trB and phosphorylation. With ATP, RcN trC ${ }^{\mathrm{C} 7}$ stimulated transcription at the nifA 1Mutl promoter $\sim 10$-fold over basal levels (Fig. 5A, Ianes 1,2). When A M P-PN P was substituted for ATP in the transcription reactions, no activation of the nifA1Mut1 promoter over the basal levels was observed (Fig. 5, lanes 3,4).

The result with AM P-PN P could be caused by either a requirement for ATP hydrolysis or an inability of RcN trC to bind AMP-PN P. In fact, transcriptional activation by the enteric N trC (S. Kustu, pers. comm.) and the EBP, DctD (T.R. Hoover, pers. comm.), is not inhibited by AMP-PNP. This is in contrast to ADP and ATP $\mathrm{S}$ that both inhibit activation by enteric N trC (Popham et al . 1989; Weiss et al. 1991), suggesting that AM PPNP may not bind these EBPs. Similarly, we tested whether AMP-PN P, ADP, and ATP $\gamma$ S inhibit the ATPdependent activation property of RcN trC. N one of the anal ogs inhibited activation, suggesting that they do not bind RcN trC, or alternatively, they bind and can function as coactivators (Fig. 5B shows the result with ADP). To resolve this, in place of ATP, we initially tested ATP $y$ S, which can be incorporated into RN A but is considered nonhydrolyzable. Reactions with ATPyS resulted in at least a 10-fold increase in transcription in the presence of RCN trC (Fig. 5C, lane 2) compared with basal levels (lane 1). Again, ADP did not inhibit this activation (Fig. 5C, cf. lane 4 with lane 3), even at ADP levels 30- fold higher then ATP $\gamma$ S. To confirm that ADP does not act as a coactivator, ADP was added with AM P-PN P and results identical to those shown in Figure $5 A$ were observed (data not shown). Taken together, we conclude that, in addition to phosphorylation by $\mathrm{N} \operatorname{trB}, \mathrm{RcN} \operatorname{trC}$ requires the specific binding of ATP for transcriptional activation.

The enteric RNAP and RcNtrC activation

We have been unsuccessful in attempts to find condi-

A

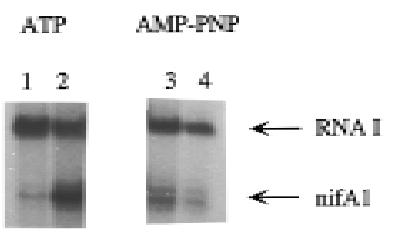

B

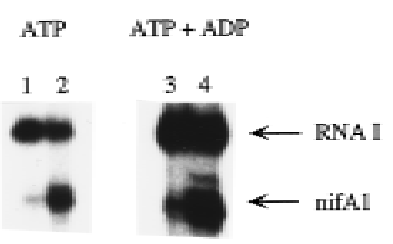

C

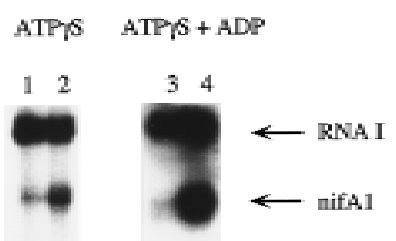

Figure 5. Effects of ADP and nonhydrolyzable ATP analogs on transcriptional activation by RcN trC. For each set of reactions, lanes 1 and 3 contained no activator and lanes 2 and 4 contained $350 \mathrm{~nm} \mathrm{RcN} \operatorname{trC}^{\mathrm{C} 7}$. (A) In vitro transcription reactions were performed using either ATP (lanes 1,2) or AM P-PN P (Ianes 3,4) at $1.5 \mathrm{~mm}$. (B) In vitro transcription reactions were performed in the presence of $1.5 \mathrm{~mm}$ ATP (lanes 1,2) or $0.4 \mathrm{~mm}$ ATP and 15 mM ADP (lanes 3,4). (C) In vitro transcription reactions were performed in the presence of $1.5 \mathrm{~mm}$ ATP $\gamma \mathrm{S}$ (lanes 1,2$)$ or 0.4 $\mathrm{mm}$ ATP $\gamma \mathrm{S}$ and $15 \mathrm{~mm}$ ADP (lanes 3,4). 
tions in which the E. coli RNAP $\sigma^{70}$ is activated by RcN trC at wild-type or nifA1Mutl promoters (e.g., see Fig. 6, lanes 4-6). The E. coli RN AP was also not activated when the purified enteric $\sigma^{54}$ subunit was added to supercoiled templates containing the wild-type or nifA 1Mut1 promoters. This later result with $\sigma^{54}$ is not unexpected because none of the RcN trC-dependent promoters are $\sigma^{54}$-type (i.e., GG-N 10-GC). The failure of RcN trC to activate the E. coli $\sigma^{70}$ RN AP could be the result of alack of interaction with RcN trC or inability to form an open complex at this promoter (or both). To begin to address this, we used the nifA 1Mut3 promoter, which yields high basal transcription with the RCRN AP holoenzyme. Surprisingly, the nifA 1Mut3 promoter on a supercoiled template was transcribed by E. coli RN AP at a very low basal level, at least 20 -fold less than the RNAI promoter and 50 -fold less than the R. capsulatus housekeeping enzyme (Fig. 6, lane 7). This result suggests that the $E$. coli enzyme cannot melt the -10 region of the nifA 1 promoter as the R. capsulatus enzyme can. $\mathrm{Nev}$ ertheless, a basal level of transcription with nifA 1Mut3 is observed, and this was not increased on addition of $\mathrm{RcN}$ trC and MBP-N trB relative to the RN Al transcript (Fig. 6, lanes 7-9) (see Discussion).

\section{Discussion}

RcNtrC-activated promoters

During the last 7 years, there has been considerable speculation on the promoters activated by the EBP $\mathrm{RcN} \operatorname{trC}$, and on the holoenzyme(s) that is used (e.g., Kranz and Foster-Hartnett 1990; Morett and Segovia 1993; Foster-Hartnett et al. 1994; Kranz and Cullen 1995; Masepohl and Klipp 1996). In the present study, of the four promoters tested, the -35 hexamer of the nifA1Mut1 promoter is the optimal RcNtrC-activated -35 region. We note that hexamers containing at least four out of six of this optimal sequence are present in each of the five natural RCN trC-activated promoters (Fig. 7). As in E. coli $\sigma^{70}$-activated systems (Busby and Ebright 1994), evolution away from the optimal recognition ele-

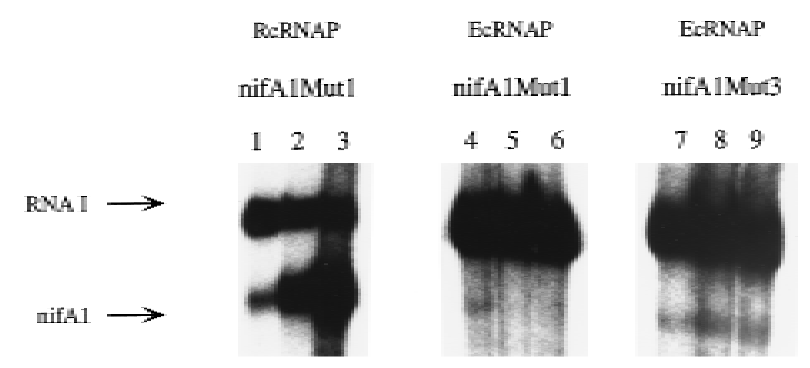

Figure 6. RcN trC does not activate the E. coli RNAP. In vitro transcription reactions were performed with purified RN AP $\sigma^{70}$ from R. capsulatus and $\mathrm{E}$. coli and the MBP-N trB/RcN trC ${ }^{C 7}$ transcriptional activation system. Templates: nifA 1Mut1 (lanes 1-6) and nifA1Mut3 (lanes 7-9). (Lanes 1,4,7) No activator; (lanes 2,5,8) 350 nm RcN trC ${ }^{\text {C7 }}$; (lanes 3,6,9) 350 nM RcN trC ${ }^{\text {C7 }}$ and $270 \mathrm{~nm}$ MBP-N trB. ment results in lower basal levels of expression, as demonstrated here for nifA 1 . Whereas a consensus -10 regi on for these promoters remains to be determined, 15- to 18-bp downstream of each of these -35 elements is a potential -10 hexamer with a second position $A$ that is $76 \%$ conserved in $\mathrm{E}$. coli $\sigma^{70}$ promoters (see Lisser and $M$ argalit 1993). All five -10 regions, including the nifA 1 promoter, also contain a GC or a GG dinucl eotide. Even if the -10 hexamers shown in Figure 7 have been incorrectly chosen, extended -10 regions show at least $50 \%$ GC content. Because the genomic GC content of R. capsulatus is $65 \%$, the $R$. capsulatus housekeeping hol oenzyme may have evolved the ability to melt high GC regions that the E. coli holoenzyme cannot. This ability is probably not limited to RcN trC-activated promoters, because other natural R. capsulatus promoters with high GC content in the -10 region are poorly transcribed by the E. coli enzyme (Cullen et al. 1997). However, this capability does not reside in the R. capsulatus $\sigma^{70}$ regions called 2.4 or 2.5 that have been implicated in melting the -10 hexamer (see M al hotra and Severinova 1996) and extended -10 bp (Barne et al. 1997), respectively; the R. capsulatus sequence of $\sigma^{70}$ is identical to that of $E$. coli in these regions (Pasternak et al. 1996).

Requirements of upstream tandem binding sites: How does RcNtrC contact RNAP?

We have demonstrated that two RcN trC dimers bound to the upstream tandem sites are necessary for activation. Phosphorylated RcN trC at $875 \mathrm{~nm}$ concentration was still unable to activate transcription from supercoiled templates with only one dimer binding site. This result is similar to that initially demonstrated for the $E$. coli N trC EBP (Ninfa et al. 1987). In the R. capsulatus system, as low as $160 \mathrm{~nm}$ unphosphorylated RcNtrC (Foster-Hartnett et al. 1994) and 80 nm of phosphorylated RcN trC was able to protect the single site from DNA se on linear DN A. Moreover, as low as 80 nm of phosphorylated RcN trC could activate transcription of nifA 1 on the templ ate containing tandem sites. These results suggest that oligomerization of at least two dimers is required for transcriptional activation. We also note conserved phasing of the tandem sites for all five RcN trCactivated promoters with either 5, 6, or 15 bp separating each RcN trC binding site (see Fig. 7). Such phasing was shown to be necessary for the EBP XyIR (Perez-Martin and de Lorenzo 1996).

All five promoters contain tandem sites that bind RcN trC located distant from the promoter. We consider three possible mechanisms by which RcN trC might contact RNAP. (1) It is possible that the DNA between RNAP and RcN trC binding sites loops out naturally as is the case for the glnA promoter and enteric $\mathrm{N}$ trC (Su et al. 1990; Wedel et al . 1990). Magasanik and colleagues have reported elegant studies recently on promoters activated by the enteric $\mathrm{NtrC}$, demonstrating that some contain natural curvature of the DNA that is necessary to optimize contact with RNAP (Carmona and Magasanik 


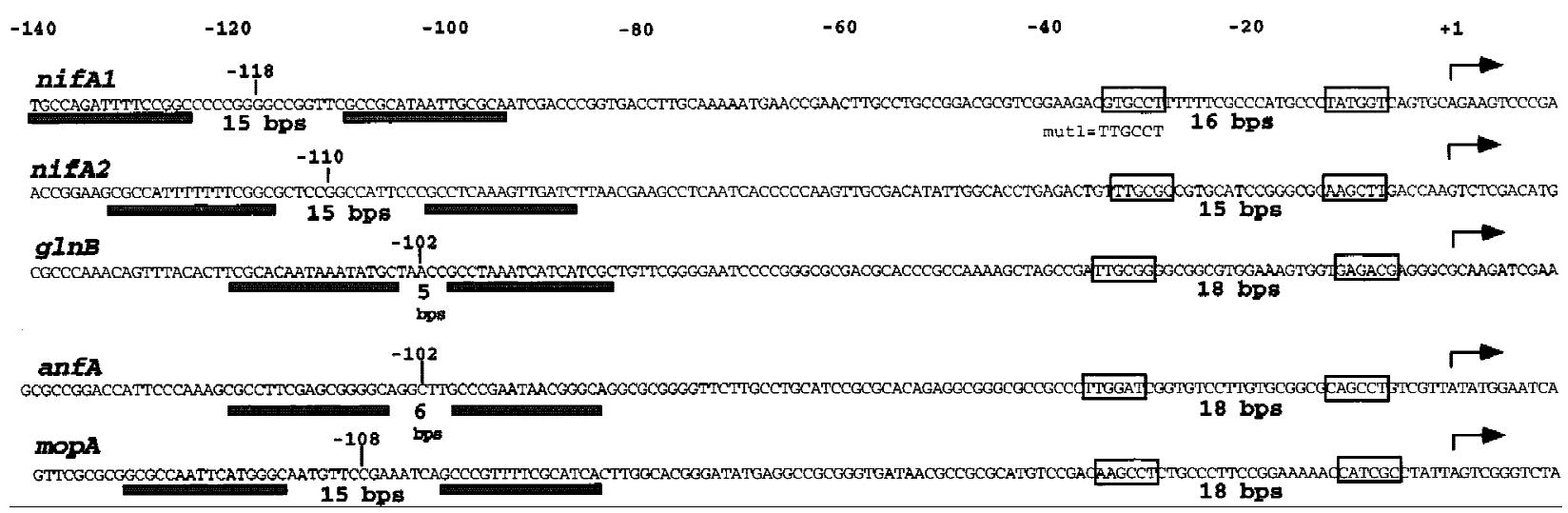

Figure 7. The five natural promoters activated by RcN trC. (Arrows) In vivo mRN A start sites. Each member of the RcN trC regul on has tandem sites containing the consensus RcN trC binding region underlined with dark boxes (Foster-Hartnett and Kranz 1994). The nifA 1 and nifA 2 tandem sites were shown previously to bind RcN trC in vitro (Foster-Hartnett et al. 1994) as were the glnB (FosterHartnett and Kranz 1994), anfA, and mopA sites (W.C. Bowman and R.G. Kranz, unpubl.). The RcN trC consensus binding region has been presented previously (Foster-Hartnett and Kranz 1994). The boxed DN A on the left of each promoter region contains at least four of the optimal -35 hexamer (M ut1) sequence. The boxed hexamers (right) are putative -10 regions discussed in the text.

1996; Carmona et al. 1997). (2) Some of the RNAP/ $\sigma^{54}$ systems require that IHF bind and loop the DNA between the promoter and EBP binding sites, thereby increasing the frequency of contact between EBP and RNAP (e.g., Santero et al. 1992). We have shown previously that IHF does not bind to the nifAI DNA (FosterHartnett et al. 1994) and no such potential IHF binding sites are found upstream of any of these promoters (Fig. 7). The RcN trC and RNAP preparations used here are $>95 \%$ pure, although we cannot rule out a minor contaminating factor that plays such a role. (3) It is possible that RcN trC multimerizes on the DNA from the upstream tandem binding sites toward the RNAP. This could involve less specific binding to DNA, particularly because no RcN trC-consensus binding sites are present in the intervening DNA between the tandem sites and promoter. We have not observed such binding on linear templates. We have engineered nine different DNA inserts of sizes from 4 to 130 bp into positions -84 and -47 , relative to the transcript start site of nifA 1 (Foster-Hartnett et al. 1994). All nine inserts resulted in loss of activation in vivo by $\mathrm{RcN}$ trC. This result indicates that the structure and/ or sequence of the intervening DNA is important, as might be expected for mechanisms (1) or (2).

The mechanism by which RcNtrC activates open complex formation by the R. capsulatus holoenzyme and the protein-protein contacts that are made, remain important questions. It is intriguing that the $\mathrm{E}$. coli enzyme is not activated at this promoter. Even with a -35 hexamer of the nifA 1 promoter (i.e., M ut3), which yields a low level of basal transcription with the E. coli holoenzyme, it cannot be further activated by RcN trC. It is therefore possible that RcN trC does not interact with the E. coli hol oenzyme. In a similar line of experiments, the Salmonella $\mathrm{NtrC}^{\mathrm{S160F}}$ protein did not activate RCRNAP (not shown) even at concentrations in which DN A binding of $\mathrm{N} \mathrm{trC}{ }^{\mathrm{S16OF}}$ is not necessary for activation of the enteric $\sigma^{54}$ RNAP (Weiss et al. 1991). These results suggest that the RcNtrC and RcRNAP may have co- evolved specific sites for interaction, although more studies will be necessary to confirm this.

\section{Requirement for ATP binding but not ATP hydrolysis}

We used the in vitro activation system to determine whether ATP binding or ATP hydrolysis is required by RcN trC. Inhibition with various ATP analogs of the ATP-dependent activation by RcN trC was analyzed. The ability of anal ogs to act as coactivators was investigated, as has been carried out with the enteric EBPs (Popham et al. 1991; Weiss et al. 1991). The results indicate that AMP-PNP and ADP do not bind RcN trC. Importantly, the nonhydrolyzable analog ATP $\gamma S$ supports activation by RcN trC (Fig. 5C). Because the nonhydrol yzable AM PPN P does not support activation when it repl aces ATP in the transcription reaction, it can also be concluded that only ATP and not other nucleotide tri phosphates are coactivators. We conclude that the RcN trC protein specifically requires ATP binding for activation, but unl ike the EBP activators of $\sigma^{54}$ RNAP, ATP hydrolysis is not essential. This is consistent with our observation that none of the purified RcN trC proteins, wild-type or constitutive, exhibit a detectable ATPase activity, with or without DNA containing tandem binding sites (P.J. Cullen and R.G. Kranz, unpubl.).

Beside the EBP family of $\sigma^{54}$ RNAP activators, two other bacterial regulators contain nucleotide binding folds and activate the $\sigma^{70}$ RN AP. TyrR binds to ATP and has an ATPase activity (Cui et al. 1993). However, mutational analysis of Walker motif A indicates that ATP binding is not necessary for its activation function (Pittard and Davidson 1991; Yang et al. 1993). The TyrR protein appears to bind between -35- and -80-bp upstream of the transcription start site when it is an activator (Wilson et al. 1994; Gralla and Collado-Vides 1996). MalT binds to both maltotriose and ATP as coactivators (Richet and Raibaud 1989). MalT always binds to the promoters that it activates near -38-bp upstream 
of the start site (Danot and Raibaud 1994) and this binding only occurs when the coactivators are present (Richet and Raibaud 1989). It is quite clear that the $\mathrm{RcN}$ trC protein binds to its upstream tandem sites in the absence of ATP, indicating that the ATP-mediated mechanism for activation will be different from that of MalT . RcN trC wild-type, RcN trC with mutations in the nucleoti de binding fold, and $\mathrm{RcN} \operatorname{trC}^{\mathrm{C} 3}$ and $\mathrm{RcN} \mathrm{trC}^{\mathrm{C} 7}$ all bind to the upstream tandem sites with similar affinities in the presence or absence of ATP (W.C. Bowman and R.G. Kranz, unpubl.). We suggest that the RcN trC protein simultaneously senses two states of the cell. One is the nitrogen status that is mediated by the classic twocomponent N trB kinase pathway (via GInB). A second is the concentration of ATP or energy status. This may not be too surprising because it is crucial that a photosynthetic bacterium knows the levels of ATP that are available for nitrogen fixation, for example, before it induces the $>36$ genes necessary for this energy-intense process (Kranz and Cullen 1995).

\section{Evolutionary aspects}

Two important properties that are essential for $\sigma^{54} /$ EBP systems are retained by the RcN trC system: (1) cooperative binding to tandem sites upstream to induce oligomerization and (2) nucl eotide binding fold in the central domain that requires ATP for activation. It is shown that the natural holoenzyme used for activation by $\mathrm{RcN}$ trC is the housekeeping RN AP and a -35 consensus region is now better defined for this unique system. Therefore, we conclude that $R$. capsulatus has three general types of activation systems: (1) the traditional $\sigma^{70}$-type in which activators bind the DNA adjacent to the RNAP (Bauer 1995; Cullen et al. 1997), (2) a bonafide $\sigma^{54}$ RN AP and EBP activators (e.g., The NifA and AnfA proteins) (Cullen et al. 1994; Kutsche et al. 1996), and (3) the RcN trC system defined here with properties of 1 and 2. Because R. capsulatus is consi dered to be one of the most metabolically versatile microorganisms, it is possible that it has evolved these systems to add to its control repertoire (i.e., for regulatory versatility). It is worth considering that a system like the RcN trC activator pathway evol ved into the classi c $\sigma^{54} /$ EBP and was retai ned by this $\alpha$ proteobacterium, along with the classical system.

\section{Materials and methods}

\section{Plasmids}

pUct-nifA 1, pA 1M 1, pA 1M 2, and pA 1M 3 have been described previously (Cullen et al. 1997). pALB1 was made by PCR of the nifA 1Mut1 promoter region in plasmid PAIM 1 with the upstream oligonucleotide 5'-CCCGGTACCGGTTCGCCGCATAATTG-3' and the downstream oligonucleotide 5'-TGACCGGCAGCAAAATG-3'. The $0.3-k b$ product was digested with Kpnl and HindlII and cloned into pU C118. pALB2 was made by PCR of the nifA 1Mutl promoter region in plasmid pA $1 M 1$ by the upstream oligonucleotide 5'-CCCGGTACCCTTGCAAAAATGAACC-3' and the same downstream oligonucleotide as pALB1. The $0.3-\mathrm{kb}$ product was digested with $\mathrm{Kpnl}$ and HindlII and cloned into pU C118. pALB1 and pALB2 were sequenced to confirm the removal of 1 or both RcN trC binding sites, respectively. Plasmid pRGK300, which allows overexpression of the R. capsulatus $\sigma^{70}$ protein, was made by PCR of the rpoD gene (Pasternak et al. 1996) from the R. capsulatus chromosome by the upstream oligonucleotide 5'-TGCGCAGCCCCGATGCAGCCCGACGAGGAG-3' and the downstream oligonucleotide5'-GCATCTTCAGATCTTCGGGGCCTTACTGGT-3'. These oligonucleotides contain $\mathrm{Ncol}$ and Bglll sites that facilitated cloning the 2-kb PCR product downstream of the T7 promoter in pET 15 (N ovagen). Plasmid pRGK301, which allows overexpression of the R. capsulatus $\sigma^{70}$ protein that contains a hexahistidine tag, was made by PCR of the rpoD gene from the R. capsulatus chromosome by use of the upstream oligonucleo tide 5'-CGAGGAGCGCATATGGCCGCCAAGGACATC-3' and the same downstream oligonucleotide that was used for pRGK300. The 2.0-kb PCR product was digested with Ndel and Bglll and ligated in frame to the amino-terminal hexa histidine tag encoded by PET 15.

\section{RNAP purification}

Purification of RNAP from E. coli and R. capsulatus has been described previously (Cullen et al. 1997). Both heparin-agarose pure and DEAE-Sepharose pure RCRNAP holoenzyme were used in in vitro transcription reactions, as well as DEAE pure R. capsulatus core RNA polymerase. For in vitro transcription reactions that utilized the E. coli RNA polymerase EcRN AP, only the DEAE-Sepharose pure fractions were used.

\section{$\sigma^{70}$ purification}

The $\sigma^{70}$ subunit from R. capsulatus without a hexahistadinetag was overexpressed in E. coli strain BL21- $\delta$ DE3 containing pRGK 300 by the addition of $1 \mathrm{~mm}$ IPTG for $3 \mathrm{hr}$ at $37^{\circ} \mathrm{C}$. Cells were sonicated in $20 \mathrm{mls}$ of buffer $(20 \mathrm{~mm}$ Tris- $\mathrm{HCl}, 2 \mathrm{~mm}$ EDTA, at pH 8) and cell debris was removed by centrifugation at $12,000 \mathrm{~g}$ for $15 \mathrm{~min}$ at $4^{\circ} \mathrm{C}$ in a Sorvall centrifuge. The supernatant contains a major polypeptide that is not present in BL21$\delta D E 3$ containing no plasmid; this polypeptide migrates at the same size as the $\sigma^{70}$ from the R. capsulatus holoenzyme preparation (not shown). The supernatant $(0.5 \mathrm{ml})$ was run on an $8 \%$ polyacrylamide gel and the $\sigma^{70}$ protein was purified from the gel and renatured by use of the method of Gross et al. (1978).

Hexahistidine-tagged $\sigma^{70}$ subunit from R. capsulatus was overexpressed in E. coli strain BL21- $\delta$ DE3 containing pRGK301 by the addition of $1 \mathrm{~mm}$ IPTG for $3 \mathrm{hr}$ at $37^{\circ} \mathrm{C}$. Cells were sonicated in $20 \mathrm{mls}$ of buffer $(20 \mathrm{~mm}$ Tris- $\mathrm{HCl}, 2 \mathrm{~mm}$ EDTA at $\mathrm{pH} 8$ ) and cell debris was removed by centrifugation at $12,000 \mathrm{~g}$ for $15 \mathrm{~min}$ at $4^{\circ} \mathrm{C}$ in a Sorvall centrifuge. The supernatant contains a major polypeptide that is not present in BL21- $\delta$ DE3 containing no plasmid; this polypeptide was similar in size by SDSPAGE to the R. capsulatus $\sigma^{70}$ subunit in the holoenzyme preparation (not shown). The supernatant was loaded onto a His-Bind (N ovagen) column and the column was washed with 10 volumes of binding buffer $(5 \mathrm{~mm}$ imidazole, $0.5 \mathrm{M} \mathrm{N} \mathrm{aCl}, 20$ $\mathrm{mm}$ Tris- $\mathrm{HCl}$ at $\mathrm{pH} 7.9$ ), followed by 10 volumes of wash buffer (60 mm imidazole, $0.5 \mathrm{M} \mathrm{N} \mathrm{aCl}, 20 \mathrm{~mm}$ Tris- $\mathrm{HCl}$ at $\mathrm{pH}$ 7.9). The histidine-tagged $\sigma^{70}$ was eluted in 6 volumes of elution buffer ( $250 \mathrm{~mm}$ imidazole, $0.5 \mathrm{M} \mathrm{N} \mathrm{aCl}, 20 \mathrm{~mm}$ Tris- $\mathrm{HCl}$ at $\mathrm{pH}$ 7.9). The protein was concentrated to $1 / 10$ of the original volume in a Centricon 30, diluted to $1 \mathrm{ml}$ with storage buffer $(50 \mathrm{~mm}$ Tris$\mathrm{HCl}, 0.5 \mathrm{~mm}$ EDTA at pH 8, $1 \mathrm{~mm}$ DTT, 50\% glycerol), and stored at $-80^{\circ} \mathrm{C}$.

\section{Purification of MBP-NtrB and RcNtrC ${ }^{C}$ proteins}

Purification of the MBP-N trB has been described previously (Cullen et al. 1996). RcN trC constitutive mutant proteins 
$\left(\mathrm{RcN}_{\operatorname{trC}} \mathrm{C}^{\mathrm{C}}\right.$ ) were purified through the DEAE-Sepharose step by use of the method of Cullen et al. (1996). The isolation of genes encoding R. capsulatus $\mathrm{N} \operatorname{trC}^{c}$ alleles will be described elsewhere.

\section{In vitro transcription}

In vitro transcription reactions were performed in transcription buffer [50 mM Tris- $\mathrm{HCl}$ at $\mathrm{pH} 8,100 \mathrm{~mm}$ potassium acetate, 10 mM magnesium acetate, $1 \mathrm{~mm}$ ATP, $10 \mathrm{~mm}$ DTT and $0.5 \mu \mathrm{l}$ RN Asin (Promega)] by the method of Cullen et al. (1997). The concentration of linear or supercoiled templates was $\sim 40 \mathrm{~nm}$ for all reactions reported here. For in vitro transcriptional activation reactions, MBP-N trB (270 nM) was incubated in transcription buffer for $10 \mathrm{~min}$ at $37^{\circ} \mathrm{C}$ prior to the addition of $\mathrm{RcN} \operatorname{trC}^{\mathrm{C}}$ (80-875 nm as noted) and RN AP (40 nM). RcN trC ${ }^{\mathrm{C}}$ and RN AP were added simultaneously and the reactions were incubated at $24^{\circ} \mathrm{C}$ for $30 \mathrm{~min}$. Purified $\sigma^{70}$ proteins were added to the core RN AP prior to the start of the reactions.

\section{DNase I footprinting}

DN ase I footprinting analysis of phosphorylated and unphosphorylated $\mathrm{RCN}_{\mathrm{trC}} \mathrm{C}$ at the nifA 1 promoter region was performed by the method of Cullen et al. (1996). Probes were prepared by digesting plasmids (pAIM 1 of pALB1) with EcoRI and dephosphorylating the ends with calf intestinal phosphatase. The $5^{\prime}$ ends were labeled with [ $\gamma^{-32}$ P]ATP and T4 polynucleotide kinase, then the labeled DNA was digested with HindlII. After separation on a $4 \%$ native acrylamide gel $(50 \mathrm{~mm}$ Trisborate EDTA), gel sliced that contained the appropriate fragments were excised and the probes were eluted overnight at $37^{\circ} \mathrm{C}$ in Tris-EDTA buffer. Approximately $0.2 \mathrm{~nm}$ of probe $(30,000-60,000 \mathrm{cpm})$ was used in each footprinting reaction.

\section{Other methods}

Western analysis was performed with peroxidase detection reagents from Pierce. Use of monoclonal antibody 2G10, a gift from $\mathrm{N}$ ancy Thompsen and Richard Burgess (University of Wisconsin, M adison) and monoclonal antibodies to $\beta$-gal actosi dase have been described previously (Cullen et al. 1997). Transcripts were quantitated by scanning the autoradiograms with an HP scanjet $4 C$ and analyzing the bands by use of IP Labgel software from Data Analysis Corp (M al ek et al. 1997). The scanning and software could easily distinguish differences in transcript levels twofold or above. Protein sequencing was carried out with an Applied Biosystems 470A protein sequencer.

\section{Acknowledgments}

We thank Paul Cullen and Charles Kaufman for the housekeeping RN AP preparation and Western blotting; Barry Goldman for the construction of pRGK300 and pRGK301 and the purification of the histidine-tagged $\sigma^{70}$ protein; Anne Bradburn for the construction of pALB1 and pALB2; Sydney Kustu and Andrea Shauger for providing $\sigma^{54}$ and $\mathrm{N}$ trC $\mathrm{C}^{\mathrm{S} 160 \mathrm{~F}}$ proteins; $\mathrm{N}$ ancy Thompsen and Richard Burgess for mAb 2G 10; Steve Slater and Richard Thomas at Monsanto for protein sequencing; Sydney Kustu for comments on the manuscript and advice on the ATPdependence function of RcN trC; Ray Dixon, Tim Hoover, Mike Merrick, Alex Ninfa for comments on the manuscript. This work was supported by U.S. Department of Agriculture $\mathrm{Na}$ tional Research Initiative grant 9703754 (to R.G.K.).

The publication costs of this article were defrayed in part by payment of page charges. This article must therefore be hereby marked "advertisement" in accordance with 18 USC section 1734 solely to indicate this fact.

\section{References}

Austin, S. and R. Dixon. 1992. The prokaryotic enhancer binding protein N trC has an ATPase activity which is phosphorylation and DNA dependent. EMBO J. 11: 2219-2228.

Barne, K.A., J.A. Bown, J.W. Busby, and S.D. Minchin. 1997. Region 2.5 of the Escherichia coli RN A polymerase sigma 70 subunit is responsible for the recognition of the 'extended -10' motif at promoters. EMBO J. 16: 4034-4040.

Bauer, C.E. 1995. Regulation of photosynthesis gene expression. In Anoxygenic photosynthetic bacteria (ed. R.E. Blankenship, M.T. Madigan, and C.E. Bauer), pp. 1221-1234. Kluwer, Dordrecht, The $\mathrm{N}$ etherlands.

Breyer, M.J., N.E. Thompson, and R.R. Burgess. 1997. Identification of the epitope for a highly cross-reactive monoclonal antibody on the major sigma factor of bacterial RNA polymerase. J. Bacteriol. 179: 1404-1408.

Buck, M. and W. Cannon. 1992. Specific binding of the transcription factor sigma-54 to promoter DNA. Nature 358: $422-424$.

Busby, S. and R.H. Ebright. 1994. Promoter structure, promoter recognition, and transcription activation in prokaryotes. Cell 79: 743-746.

Cannon, W., F. Claverie-M artin, S. Austin, and M. Buck. 1993. Core RNA polymerase assists binding of the transcription factor $\sigma^{54}$ to promoter DNA. Mol. Microbiol. 8: 287-298.

Carmona, M. and B. Magasanik. 1996. Activation of transcription at sigma 54-dependent promoters on linear templates requires intrinsic or induced bending of the DNA. J. Mol. Biol. 261: 348-356.

Carmona, M., F. Claverie-Martin, and B. Magasanik. 1997. DNA bending and the initiation of transcription at sigma 54-dependent bacterial promoters. Proc. Natl. Acad. Sci. 94: 9568-9572.

Collado-Vides, J., B. M agasanik, and J. Gralla. 1991. Control site location and transcriptional regulation in Escherichia coli. Microbiol. Rev. 55: 371-394.

Cui, J., L. Ni, and R.L. Somerville. 1993. ATPase activity of TyrR, a transcriptional regulatory protein for sigma 70 RN A polymerase. J. Biol. Chem. 268: 13023-13025.

Cullen, P.J., D. Foster-Hartnett, K. Gabbert, and R.G. Kranz. 1994. Structure and expression of the al ternative sigma factor, RpoN, in Rhodobacter capsulatus; physiological relavance of an autoactivated nifU 2-rpoN superoperon. Mol. Microbiol. 11: 51-65.

Cullen, P.J., W.C. Bowman, and R.G. Kranz. 1996. In vitro re constitution and characterization of the Rhodobacter capsulatus $\mathrm{N}$ trB and $\mathrm{N}$ trC two-componet system. J. Biol. Chem. 271: 6530-6536.

Cullen, P.J., C.K. Kaufman, W.C. Bowman, and R.G. Kranz. 1997. Characterization of the Rhodobacter capsulatus housekeeping RNA polymerase. In vitro transcription of photosynthesis and other genes. J. Biol. Chem. 272: 27266-27273.

Danot, O. and O. Raibaud. 1994. Multiple protein-DNA and protein-protein interactions are involved in transcriptional activation by MalT. Mol. Microbiol. 14: 335-346.

Foster-Hartnett, D. and R.G. Kranz. 1992. Analysis of the promoters and upstream sequences of nifA 1 and nifA 2 in Rhodobacter capsulatus; activation requires $\mathrm{N}$ trC but not RpoN. Mol. Microbiol. 6: 1049-1060.

- - - 1994. The Rhodobacter capsulatus glnB gene is regulated by $\mathrm{N}$ trC at tandem RpoN-independent promoters. J. Bacteriol. 176: 5171-5176.

Foster-Hartnett, D., P.J. Cullen, E.M. Monika, and R.G. Kranz. 1994. A new type of N trC transcriptional activator. J. Bacteriol. 176: 6175-6187. 
Gentry, D.R. and R.R. Burgess. 1986. The cloning and sequencing of the gene encoding the omega subunit of Escherichia coli RN A polymerase. Gene 48: 33-40.

Gralla, J.D. and J. Collado-Vides. 1996. Organization and function of transcription regulatory el ements. In Escherichia coli and Salmonella (ed. F.C. N eidhardt) pp. 1232-1245. ASM Press, Washington D.C.

Gross, C., J. Hoffman, C. Ward, D. Hager, G. Burdick, H. Berger, and R. Burgess. 1978. Mutation affecting thermostability of sigma subunit of Escherichia coli RN A polymerase lies near the dnaG locus at about $66 \mathrm{~min}$ on the $\mathrm{E}$. coli genetic map. Proc. Natl. Acad. Sci. 75: 427-431.

Ishihama, A. 1993. Protein-protein communication with the transcription apparatus. J. Bacteriol. 175: 2483-2489.

Jones, R. and R. Haselkorn. 1989. The DN A sequence of the Rhodobacter capsulatus ntrA, ntrB and ntrC gene analogues re quired for nitrogen fixation. Mol. \& Gen. Genet. 215: 507-516.

Kranz, R.G. and D. Foster-Hartnett. 1990. Transcriptional regulatory cascade of nitrogen-fixation genes in anoxygenic photosynthetic bacteria: Oxygen- and nitrogen-responsive factors. Mol. Microbiol. 4: 1793-1800.

Kranz, R.G. and P.J. Cullen. 1995. Regulation of nitrogen fixation genes. In Anoxygenic photosynthetic bacteria (ed. R.E. Blankenship, M. M adigan, and C.E. Bauer), pp. 1191-1208. Kluwer Academic Publishing, Dordrecht, The N etherlands.

Kutsche, M., S. Leimkuhler, S. Angermuller, and W. Klipp. 1996. Promoters controlling expression of the alternative nitrogenase and the molybdenum uptake system in Rhodobacter capsulatus are activated by $\mathrm{N}$ trC, independent of sigma 54 , and repressed by molybdenum. J. Bacteriol. 178: 2010-2017.

Lee, J.H. and T.R. Hoover. 1995. Protein crosslinking studies suggest that Rhizobium meliloti $\mathrm{C}_{4}$-dicarboxylic acid transport protein $D, a \sigma^{54}$-dependent transcriptional activator, interacts with $\sigma^{54}$ and the $\beta$ subunit of RNA polymerase. Proc. Natl. Acad. Sci. 92: 9702-9706.

Lisser, S. and H. M argalit. 1993. Compliation of E. coli mRN A promoter sequences. Nucleic Acids Res. 21: 1507-1516.

Lonetto, M., M.C. Gribskov, and C. Gross. 1992. The $\sigma^{70}$ family: Sequence conservation and evolutionary relationships. J. Bacteriol. 174: 3843-3849.

Magasanik, B. 1996. Regulation of nitrogen utilization. In Escherichia coli and Salmonella (ed. F.C. N eidhardt), pp. 13441356. ASM Press, Washington D.C.

Malek, A.M., S. Izumo, and S.L. Alper. 1997. Quantitative densitometric analysis using a commercially available handheld cd digital camera. BioTechniques 22: 1150-1153.

Malhotra, A. and E. Severinova. 1996. Crystal structure of a sigma 70 subunit fragment from E. coli RNA polymerase. Cell 87: 127-136.

M asepohl, B. and W. Klipp. 1996. Organization and regulation of genes encoding the molybdenum nitrogenase and the alternative nitrogenase in Rhodobacter capsulatus.Arch. Microbiol. 165: 80-90.

Merrick, M.J. 1993. In a class of its own-the RNA polymerase sigma factor sigma 54 (sigma N ). Mol. Microbiol. 10: 903-909.

Morett, E. and L. Segovia. 1993. The $\sigma^{54}$ bacterial enhancerbinding protein family: Mechanism of action and phyloge netic relationship of their functional domains. J. Bacteriol. 175: 6067-6074.

Ninfa, A.J. and B. Magasanik. 1986. Covalent modification of the glnG product, NRI, by the gI $\mathrm{nL}$ product, NRII, regulates the transcription of the glnALG operon in Escherichia coli. Proc. Natl. Acad. Sci. 83: 5909-5913.

Ninfa, A.J., L.J. Reitzer, and B. Magasanik. 1987. Initiation of transcription at the bacterial glnAp2 promoter by purified E. coli components is facilitated by enhancers. Cell 50: 1039-1046.
N orth, A.K., K.E. Klose, K.M. Stedman, and S. Kustu. 1993. Prokaryotic enhancer-binding proteins reflect eukaryotelike modularity: The puzzle of nitrogen regulatory protein $C$. J. Bacteriol. 175: 4267-4273.

Pasternak, C., W. Chen, C. Heck, and G. Klug. 1996. Cloning, nucleotide sequence and characterization of the rpoD gene encoding the primary sigma factor of Rhodobacter capsulauts. Gene 176: 177-184.

Perez-M artin, J. and V. de Lorenzo. 1996. ATP binding to the sigma 54-dependent Activator XyIR triggers a protein multimerization cycle catalyzed by UAS DNA. Cell 86: 331339.

Pittard, A.J. and B.E. Davidson. 1991. TyrR protein of Escherichia coli and its role as repressor and activator. Mol. Microbiol. 5: 1585-1592.

Popham, D.L., D. Szeto, J. Keener, and S. Kustu. 1989. Function of a bacterial activator protein that binds to transcriptional enhancers. Science 243: 629-635.

Popham, D., J. Keener, and S. Kustu. 1991. Purification of the al ternative $\sigma$ factor, $\sigma^{54}$, from Salmonella typhimurium and characterization of the $\sigma^{54}$-holoenzyme. J. Bio. Chem. 256: 19510-19518.

Preker, P., P. Hübner, M. Schmehl, W. Klipp, and T.A. Bickle. 1992. Mapping and characterization of the promoter elements of the regulatory nif genes rpoN, nifA 1 and nifA 2 in Rhodobacter capsulatus. Mol. Microbiol. 6: 1035-1048.

Richet, E. and O. Rai baud. 1989. MalT, the regulatory protein of the Escherichia coli maltose system, is an ATP-dependant transcriptional activator. EMBO J. 8: 981-987.

Santero, E., T. Hoover, A. N orth, D. Berger, S. Porter, and S. Kustu. 1992. Role of integration host factor in stimulating transcription from the $\sigma^{54}$-dependent nifH promoter. J. Mol. Biol. 227: 602-620.

Sasse-D wight, S. and J.D. Gralla. 1988. Probing the Escherichia coli glnALG upstream activation mechanism in vivo. Proc. Natl. Acad. Sci. 85: 8934-8938.

Scott, J.R. 1984. Regulation of plasmid replication. Microbiol. Rev. 48: 1-23.

Su, W., S. Porter, S. Kustu, and H. Echols. 1990. DN A-looping and enhancer activity: Association between DNA-bound $\mathrm{N}$ trC activator and RNA polymerase at the bacterial glnA promoter. Proc. Natl. Acad. Sci. 87: 5504-5508.

Syed, A. and J.D. Gralla. 1997. Isolation and properties of enahncer-bypass mutants of sigma 54. Mol. Microbiol. 23: 987-995.

Wang, J.T., A. Syed, M. Hsieh, and J.D. Gralla. 1995. Converting Escherichia coli RN A polymerase into an enhancer-responsive enzyme: Role of an amino-terminal leucine patch in sigma 54. Science 270: 992-994.

Wedel, A., D.S. Weiss, D. Popham, P. Droge, and S. Kustu. 1990. A bacterial enhancer functions to tether a transcriptional activator near a promoter. Science 248: 486-490.

Weiss, D.S., J. Batut, K.E. Klose, J. Keener, and S. Kustu. 1991. The phosphorylated form of the enhancer-binding protein NTRC has an ATPase activity that is essential for activation of transcription. Cell 67: 155-167.

Wilson, T.J., P. Maroudas, G.J. Howlett, and B.E. Davidson. 1994. Ligand-induced self-association of the Escherichia coli regulatory protein TyrR. J. Mol. Biol. 238: 309-318.

Wyman, C., I. Rombel, A.K. N orth, C. Bustamante, and S. Kustu. 1997. Unusual oligomerization required for activity of $\mathrm{NtrC}$, a bacterial enhancer-binding protein. Science 275: 1658-1661.

Yang, J., S. Ganesan, J. Sarsero, and A.J. Pittard. 1993. A genetic analysis of various functions of the TyrR protein of Escherichia coli. J. Bacteriol. 175: 1767-1776. 


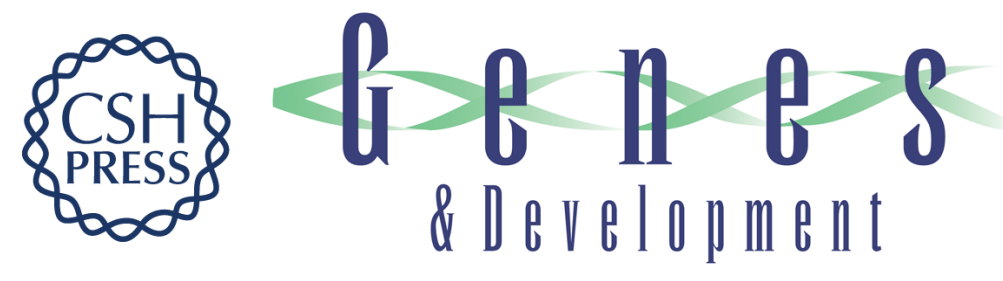

\section{A bacterial ATP-dependent, enhancer binding protein that activates the housekeeping RNA polymerase}

William C. Bowman and Robert G. Kranz

Genes Dev. 1998, 12:

Access the most recent version at doi:10.1101/gad.12.12.1884

References This article cites 52 articles, 25 of which can be accessed free at: http://genesdev.cshlp.org/content/12/12/1884.full.html\#ref-list-1

License

Email Alerting

Receive free email alerts when new articles cite this article - sign up in the box at the top Service right corner of the article or click here.

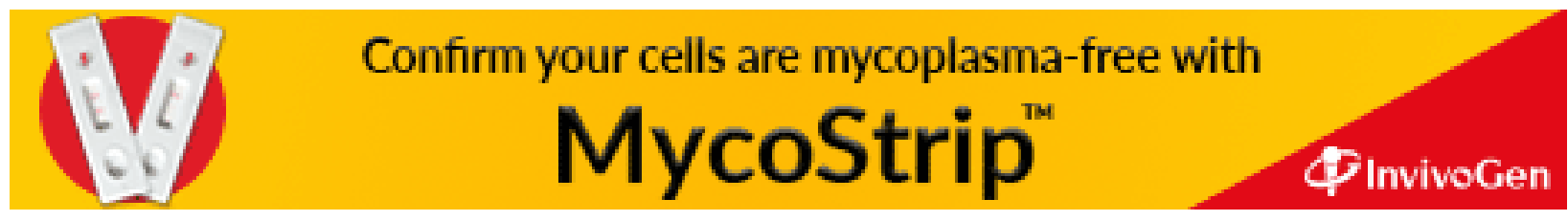

